\title{
MINI ENVIRONMENT CONTROL FOR THE ELLIPTICAL POLARIZATION UNDULATOR
}

\author{
D. S. Lee, Z. D. Tsai and J. R. Chen ${ }^{*}$ \\ SRRC, Hsinchu, Taiwan, R.O.C. \\ *National Tsing-Hua University, Department of Nuclear Science, Taiwan, R.O.C.
}

\begin{abstract}
The elliptical polarization undulator (EPU) has been constructed at SRRC for creating a high quality and high photon flux. The EPU consists of two fixed diagonal magnet arrays and the other free diagonal arrays to create the right/left circular polarization. The magnetic field uniformity of the magnet blocks will influence on the storage beam orbit, the beam life time, light source quality and brightness and it's very important to shim the magnet blocks for the magnetic field control. The environmental temperature variation in the storage ring will deform the EPU structure and change the clearance between the diagonal magnet arrays. The deformation will influence on EPU performance and impinge on the beam stability. The mini environment design concept will be employed to eliminate the EPU surrounding temperature fluctuation. By the mini environment air conditioning design, the temperature variation of the four EPU magnet arrays during 4 days operation can be limited in less than $0.1 \mathrm{deg}$ C. The temperature distribution fluctuation can be reduced to $0.22 \mathrm{deg} \mathrm{C}$
\end{abstract}

\section{INTRODUCTION}

The elliptical polarization undulator is configured by longitudinally dividing the upper and lower magnetic arrays. Such device can simultaneously generate horizontal and vertical on-axis magnetic fields by longitudinal translation of one pair of its diagonal magnetic arrays in relation to the other pair of diagonal arrays. Synchrotron Radiation Research Center (SRRC) has already constructed a 3.9 meter long Sasaki type elliptically polarizing undulator with $5.6 \mathrm{~cm}$ magnetic period length, which is optimized to provide a highly polarizing light in the 80 to $1500 \mathrm{KeV}$ range. [1]

The insertion device can create a high quality and high photon flux light only when the field shimming was done. That means the magnet block should be very careful to be assembled on the keeper to avoid the mechanical error. Each magnet blocks were glued on the individual holder by the proxy. Meanwhile, the magnet block was fixed on the holder by the clamp [2]. Such precision assemble work can achieve the high mechanical accuracy. Using the laser interferometer and dial gauge in different phase position and gap to measure the mechanical alignment. The $1 \mathrm{~mm}$ gap variation between two arrays on the lower/upper blocks can be maintained within +/- 20 um. The reproducibility of the phasing and gap moving can be controlled within $+/-1 \mathrm{um}$. It is the excellent work for the mechanical alignment. However, the temperature difference will induce the mechanical distortion error like the sag distortion of the backing beam will be 0.03 $\mathrm{mm} / \mathrm{deg} \mathrm{C}-\mathrm{m}$, it is a significant amount to induce the field error for a long undulator. According to the above measurement results, the EPU environment temperature shall be well controlled to reduce the thermal deformation of the EPU structure.

The air conditioning for the EPU area is integrated within the central system. That means the control feedback covers the whole storage tunnel air temperature variation and the control system can't response to the local EPU area temperature variation.

Under this limitation, the EPU environmental temperature can't be controlled well by the control parameter tune in. The alternative method is to isolate the EPU area to achieve the well uniform temperature control. Such isolation called mini environment is extensively employed for the cleanness control and constant temperature and humidity control. The details will be described in the later sections.

\section{EXPERIMENT FACILITY}

\subsection{Air Conditioning System for the Storage Ring}

The air conditioning system for the storage ring in SRRC consists of four air conditioning units. The air circulation condition can be referred to the figure 1 . After the return air flow are mixed by the fresh air, flow will pass through the cold heat exchanger and be cooled down to $12 \sim 13$ deg C. It also will dehumidify the moisture of the air. Then the air flow will be heated up by the hot heat exchanger and delivered to the storage ring tunnel.

The temperature control of the storage ring is achieved by controlling the hot water flow rate through the hot heat exchanger. The control feedback signal is the temperature sensor located neat the return air exhaust. Since four air handling units were employed for the whole system, one control feedback sensor covers the one forth section temperature variation message and can't well control the localization area temperature.

\subsection{Mini Environment for the EPU Area}

Isolating the EPU area and modifying the air delivering system are the only solution to achieve the accuracy local 
area temperature control. Such modification called mini environment is due to the isolation of the small EPU area. The insertion device was surround by the transparent canvas supported by the steel bar structure. Figure 2 shows the isolation area photograph.

Except isolating the EPU area, the air delivering system was also modified to meet the precision control demand. The modified structure is shown in the figure 3. According to the figure, one small air handling unit with $6000 \mathrm{cfm}$ flow rate was employed for air delivering. The EPU device is located in the end of the air conditioning wind duct system so the new air handling unit can connect to the original wind duct to delivery air. One shutter was assembled in the connection joint of the wind duct to throttle the air flow delivered by the storage ring central air conditioning system.

The added air handling unit for EPU isolation area air conditioning sucks air from the experiment area. The air temperature setting of the experiment area is lower than the storage ring tunnel by $2 \mathrm{deg}$ C. So one $6 \mathrm{~kW}$ electric heater were employed to heat up the air from experiment area. By the heating control, the EPU area can be kept in constant temperature

Since the draft of EPU area is from outside area by another air delivering system. The area can be kept in positive pressure and against the temperature variation in the other storage ring area. The surrounding temperature variation can be limited in $+/-0.1 \mathrm{deg} \mathrm{C}$ during 4 days operation except the beam shut down period. Before the modification, the EPU area temperature control can only be kept in +/- $0.25 \mathrm{deg} \mathrm{C}$ level. The comparison can be referred to figure 4 .

\subsection{The Precision Temperature Control Effect on Beamline Stability}

The EPU area temperature variation will impact the magnet arrays structure and cause the beamline position fluctuate. The correlation can be proved by the following test. As the beamline global feedback on and the EPU gap was fixed in $20 \mathrm{~mm}$, the EPU area temperature was rose up $1 \operatorname{deg} \mathrm{C}$ immediately by the electric heater. The variation can be demonstrated as the impulse function input and the beam position monitor downstream the EPU shows the $2.1 \mathrm{um}$ shift in $\mathrm{X}$ direction and $0.8 \mathrm{um}$ shift in $\mathrm{Y}$ direction. The time history of the beam position and the EPU area temperature can be referred to figure 5. Since the EPU gap was fixed, the beamline position shift was fully caused by the thermal deformation due to the EPU area air conditioning temperature variation. Assume the correlation is the linear dependent, the $+/-0.1 \mathrm{deg} \mathrm{C}$ temperature control can reduce the beam position shift by thermal impact to $0.2 \mathrm{um}$.

\subsection{The Uniformity of the EPU Magnet Arrays}

The temperature distribution of EPU magnet arrays can cause the whole structure tilt. How to uniform the temperature on the magnet arrays is another problem for the EPU temperature control.

To uniform the temperature on the EPU magnet arrays, five cross flow fans were employed to circulate the air around the magnet arrays. Four small fan units were located on the top and in front of the EPU device. One large fan was in the bottom and the back position. The air flow form the air handling unit can be draft down by the top fans and enwrap the EPU magnet arrays.

By the circulation forced convection cooling, the EPU magnet arrays temperature variation of the top magnet arrays can be reduced to $0.24 \mathrm{deg} \mathrm{C}$. The bottom magnet temperature variation can be reduced to $0.20 \mathrm{deg} \mathrm{C}$. The temperature variation correspond to the $28.08 \mathrm{um}$ deformation of the top magnet arrays and $22.23 \mathrm{um}$ deformation of the bottom magnet arrays in longitudinal direction.2.10 Acronyms

Acronyms should be defined the first time they appear.

\section{CONCLUSION}

The EPU area temperature variation during long operation times exceed 96 hours can be kept in $+/-0.1$ $\operatorname{deg} \mathrm{C}$ draft by the mini environment control for EPU isolation air conditioning. The circulation air flow can uniform the temperature distribution to $0.2 \mathrm{deg} \mathrm{C}$ level. The precise temperature control can reduce the thermal effect on the EPU deformation and improve the beam stability in SRRC.

\section{REFERENCES}

[1] C.H. Chang, C.S. Hwang, T.C. Fan, K.H. Chen, K.T. Pan, F.Y. Lin, Ch. Wang, et al, "SRRC Elliptically Polarizing Undulator Prototype to Examine Mechanical Design Feasibility and Magnetic Field Performance", The $6^{\text {th }}$ Conference on Synchrotron Radiation Instrument, Himeji, Japan. Aug. 1997.

[2] C.H. Chang, H.H. Chen, T.C. Fan, Jenny Chen, C.S. Hwang, M.H Huang, K.T. Hsu, F.Y. Lin, C.D. Li, H.C. Liu, Ch. Wang, "Construction and Performance of the Elliptical Polarization Undulator EPU 5.6 in SRRC", Proceedings of the 1999 Particle Accelerator Conference, New York, 1999. 


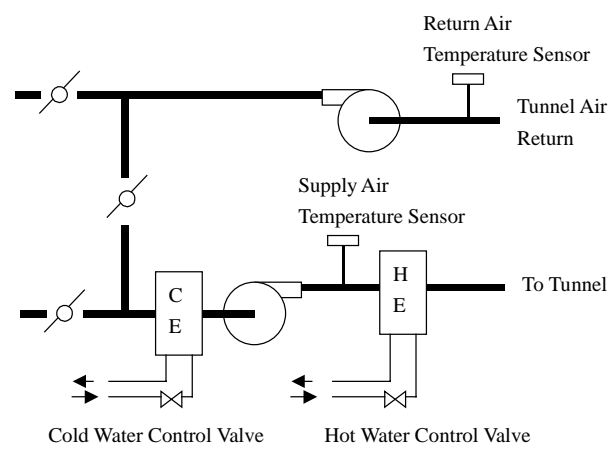

Fig 1. Central air conditioning layout

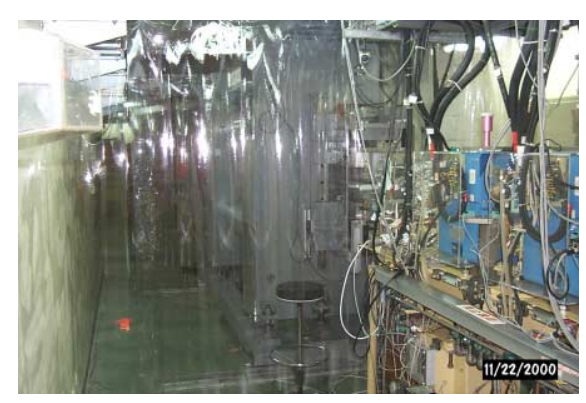

Fig 2. EPU isolation area photograph

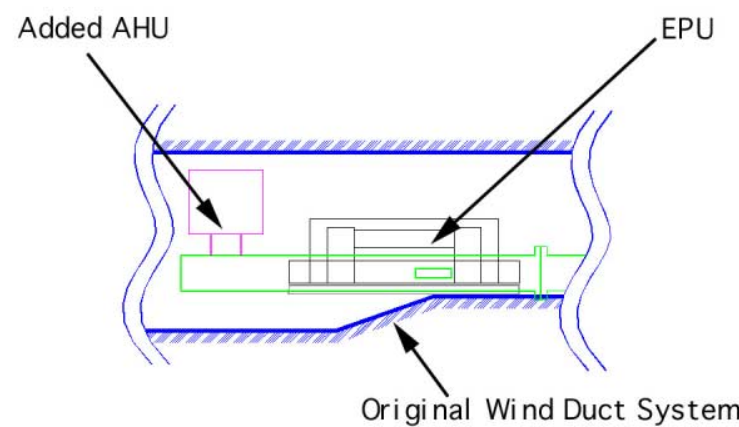

Fig 3. Mini environment modification for EPU Area
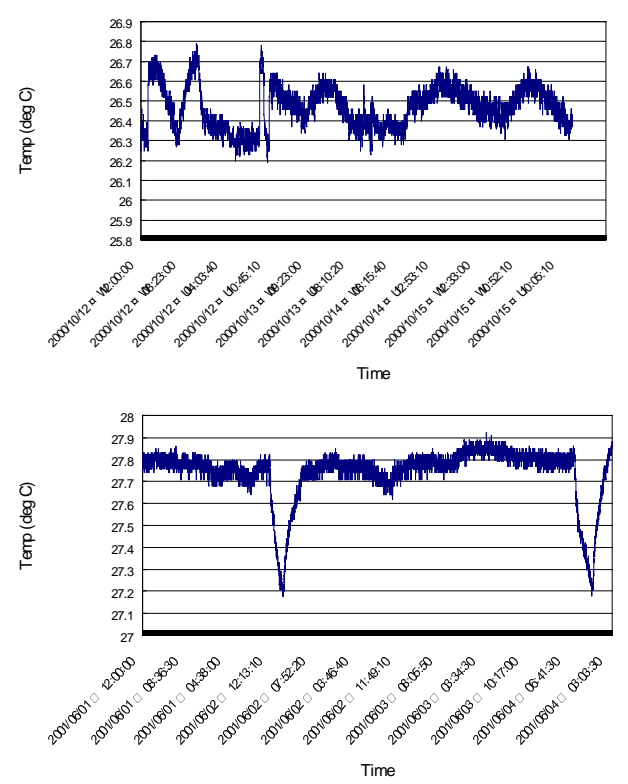

Fig 4. EPU area temperature variation in 4 days operation: Top: Central air conditioning system control Bottom: Mini environment Control
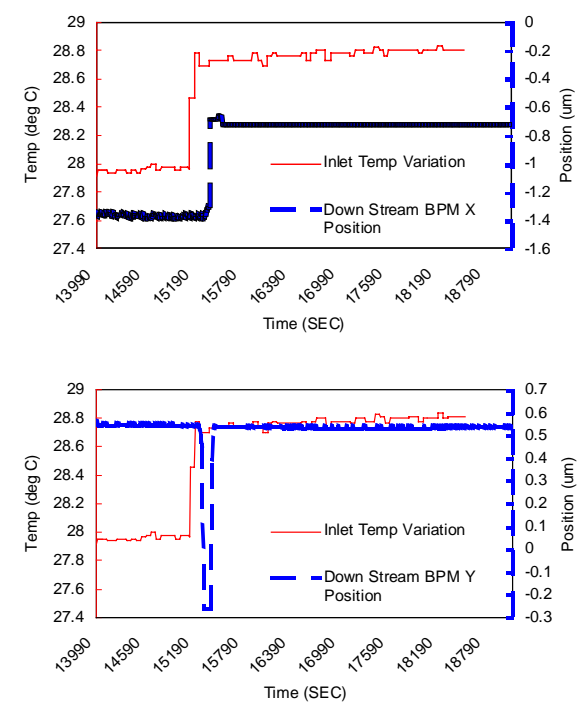

Fig 5. EPU area temperature variation and downstream beam position monitor shift 